\title{
Los cuidados en un contexto de crisis económica. El caso del área metropolitana de València
}

\author{
Care in a context of economic crisis. \\ The case of great València
}

Óscar Muñoz González • osmugon@gmail.com

UNIVERSITAT DE VALÈNCIA

ESTUDIANTE PROGRAMA DE DOCTORADO EN CIĖNCIES SOCIALS

Josep V. Pitxer i Campos · ojosep.v.pitxer@valencia.edu

UNIVERSITAT DE VALÈNCIA

DEPARTAMENT D'ECONOMIA APLICADA

Recibido: 04-04-2016

Aceptado: 18-05-2016

\section{Resumen}

La dependencia y el trabajo de cuidados se están situando en un plano central dentro del debate académico. Antaño, diversos factores incidieron en un mayor recurso a la cobertura mercantil de las necesidades crecientes en este campo, pero con la crisis económica iniciada a finales del 2007 se ha transitado hacia la desmercantilización/refamiliarización de los cuidados, consecuencia del elevado desempleo y de las políticas austericidas. La investigación descansa en la realización de once entrevistas en profundidad semiestructuradas a actores clave de la dependencia del Àrea Metropolitana de València. Se llega a la conclusión que (a) el cuidado en el ámbito doméstico es el más frecuente; (b) los principales factores para la elección de la modalidad de los cuidados son los de tipo económico y los culturales; (c) la mujer continúa como protagonista principal del cuidado en el ámbito reproductivo y es la que debe enfrentarse a la compatibilización de la vida laboral y familiar, con repercusiones negativas graves en su presencia y resultados alcanzados en el mercado de trabajo; además, (d) la falta de capacidad económica de un buen número de familias les ha conducido a abandonar el recurso al mercado, lo que, unido a la carencia de una oferta de servicios públicos suficiente, ha facilitado un desplazamiento hacia la refamiliarización del cuidado. En otros casos se han buscado alternativas mercantiles más económicas, con bastante frecuencia en contextos de informalidad, entre las que destaca la (re)nativización o retorno de la mujer autóctona a estas actividades de forma remunerada.

Palabras clave: cuidados; dependencia; conciliación vida laboral y familiar; mercado laboral.

\section{Abstract}

The dependence and care work is playing a major role in the academic debate. In the period previous to the current crisis several factors increases the market coverage of the growing needs in terms of care. However, from 2007, with the economic crisis, we have moved toward the de-commodification/refamiliarisation of care, due to high unemployment and austericity policies. The research is based on eleven in-depth semi-structured interviews with key actors of Great València. In this paper we conclude that (a) care carried on domestic sphere is the most common way to cover care needs. (b) Economic and cultural aspects are the main determinants of the type of care coverage. (c) Women are still the mainstay of care in the reproductive sphere and, due to it, they must face the conciliation between employment and family life. The result of this conflict is a negative impact on their presence and employment conditions in the labour market. In addition, (d) the shortage of monetary incomes from a large number of families explains that they were led to leave the market coverage of care. On the other hand, there is an insufficient public services supply. These two aspects have led a shift towards refamiliarisation of care work. In other cases, families have look for cheaper market alternatives, usually in a context of informality, beside the (re)nativisation or return of native women to these paid activities.

Key words: Care; Dependence; Work-life balance; Labour Market. 


\section{INTRODUCCIÓN}

La definición de dependencia viene recogida en la Ley 39/2006, de 14 de diciembre, de Promoción de la Autonomía Personal y Atención a las Personas en situación de Dependencia (en adelante, LAPAD), entendiendo como tal:

"El estado de carácter permanente en que se encuentran las personas que, por razones derivadas de la edad, la enfermedad o la discapacidad, y ligadas a la falta o a la pérdida de autonomía física, mental, intelectual o sensorial, precisan de la atención de otra u otras personas o ayudas importantes para realizar actividades básicas de la vida diaria o, en el caso de las personas con discapacidad intelectual o enfermedad mental, de otros apoyos para su autonomía personal”. (Art. 2.3 LAPAD).

El estado de dependencia citado en el texto legal no es una situación que se fije en un momento temporal-vital concreto de las personas. Niños, jóvenes, adultos o ancianos pueden hallarse en algún momento en esta situación, sea desde el nacimiento, sea como consecuencia sobrevenida de un accidente o una enfermedad, o por el mismo envejecimiento, siendo en este último donde hay mayores probabilidades de necesidad de ayuda para el desarrollo o ejercicio de las actividades básicas de la vida diaria (IMSERSO, 2005: 23). Además, las previsiones a largo plazo elaboradas por el Instituto Nacional de Estadística (INE, 2012) revelan un crecimiento de la población mayor de 65 años y un descenso en el número de personas con edades comprendidas entre los 16 y 64 años, con el consiguiente crecimiento de la tasa de dependencia.

Dada esta situación, se exige la realización de tareas para la atención y el cuidado de estas personas, con la existencia de diferentes modalidades y/o esferas de prestación. Estas actividades han sido tradicionalmente cubiertas en el seno de la unidad familiar por parte de las mujeres, derivado de la división sexual de roles en el hogar y en el trabajo. El modelo "breadwinner" de varón sustentador y mujer-esposa-ama de casa (y cuidadora) (Lewis, 1992; Sainsbury, 1994, 1996) posterior a la II Guerra Mundial es una clara muestra de esta parcelación. Pero el carácter evolutivo de la sociedad es una realidad y, hoy día, la mujer se ha incorporado al mercado laboral, lo que obliga a que esas actividades de cuidado, hasta el momento ejecutadas en la esfera privada, deban externalizarse/mercantilizarse o compatibilizarse con el empleo. No obstante, esa compatibilidad presenta sus dificultades y es frecuente hallar situaciones que responden a la etiqueta de Luis Moreno (2002) de "supermujeres" ${ }^{1 "}$ o a la doble presencia de Laura Balbo (1994), esto es, aquellas situaciones en las que las mujeres asumen una doble jornada o carga de trabajo: la propia del trabajo productivo y la del trabajo reproductivo. Junto a la incorporación de la mujer al mercado de trabajo y al crecimiento del número de personas mayores dependientes, hay un cambio en los modelos familiares o unidades de convivencia, convirtiendo a las tareas del cuidado ("care") en un elemento de debate que exige la búsqueda de soluciones.

\footnotetext{
1"Por 'supermujer' nos referimos a un tipo de mujer mediterránea que ha sido capaz de reconciliar su trabajo no remunerado en el hogar con sus cada vez mayores y más exigentes actividades profesionales en el mercado laboral formal” (Moreno, 2002: 46).
} 
Los cuidados en un contexto de crisis económica... | Óscar Muñoz González et al.

De estos menesteres puede encargarse el Estado, el mercado (a través de organizaciones de corte mercantil), el Tercer Sector o la familia, sin que sean excluyentes entre sí. Un esquema de provisión de servicios de cuidados que enlaza con el concepto de regímenes del bienestar elaborado por Esping-Andersen (1993), ampliamente estudiado, desarrollado y criticado en fechas posteriores ${ }^{2}$.

En cualquier caso, es en el ámbito de la unidad familiar donde se adoptan las decisiones fundamentales para determinar el modelo de provisión de cuidados por el que se opta, unas decisiones sujetas al grado de implicación de los poderes públicos en la cobertura de este tipo de necesidades. Dos son las decisiones familiares relevantes a este respecto. De un lado, en qué medida dichas necesidades serán atendidas en el ámbito familiar, o si por el contrario se externalizarán. De otro, cómo se distribuye la capacidad de trabajo de los integrantes de la familia entre la atención al trabajo reproductivo (el de cuidados y otros) y la dedicación al empleo. Es lo que recibe el nombre de estrategias laborales de las familias (Carbonero, 1997) o estrategias familiares de movilización laboral (Banyuls et.al. 2005: 118) y que condicionan la incorporación al mercado laboral, su abandono, o la modificación de los tiempos de trabajo productivo de los distintos miembros de las unidades familiares. Estas decisiones o estrategias diferirán de unas familias a otras según factores diversos, sin olvidar que variarán a lo largo del tiempo, entre otras cuestiones atendiendo a la situación del ciclo económico y del mercado laboral.

Con la perspectiva que acabamos de plantear, el objeto de esta investigación es analizar la visión y perspectiva profesional de un conjunto de informantes clave sobre el colectivo de personas en situación de dependencia para el caso del Àrea Metropolitana de València, así como las representaciones que desarrollan. A partir de su experiencia, se pretende: a) conocer las modalidades de prestación de servicios y la articulación y peso relativo de cada una de éstas; b) determinar los factores que condicionan la elección de uno u otro ámbito de cuidado; c) construir un marco de posibles estrategias laborales de las familias y averiguar sobre quién recae el trabajo de cuidados; y d) evaluar el impacto de la crisis sobre las estrategias de cuidados de las personas/familias cuidadoras de dependientes.

Con estos objetivos, se partió de la hipótesis de que la crisis económica española iniciada a finales del 2007 ha alentado el trabajo de cuidados en el ámbito doméstico. La falta de capacidad económica de buena parte de la población, consecuencia de la intensa destrucción de empleo, y los efectos de las políticas de austeridad impulsadas desde el año 2010 y acentuadas en el 2012, propiciaron una progresiva refamiliarización de los cuidados, espacio en el que la mujer continúa siendo la principal protagonista. Una hipótesis que supone dar continuidad a un modelo predominantemente familiar y feminizado, propio de los regímenes de bienestar mediterráneos, con servicios sociales escasos e insuficientes. A su vez, el hecho

${ }^{2}$ Pueden señalarse otras clasificaciones posibles: según el ámbito donde se efectúen los cuidados (doméstico o extradoméstico), según sea una actividad remunerada o no remunerada, o según se califiquen como formales, informales o mixtos, presentes estos últimos "cuando intervienen tanto cuidados formales como informales" (Minguela y Camacho, 2015: 7). 
de tener a una persona en situación de dependencia a su cargo provoca que las familias deban adaptarse de una u otra manera a la hora de conciliar las tareas propias del trabajo productivo/mercantil con las tareas del cuidado, emergiendo distintas estrategias laborales en el seno familiar las cuales están sujetas a diferentes condicionantes.

La estructura del texto se divide en cuatro apartados, incluyendo el actual. Un primero, de naturaleza introductoria y presentación del estado de la cuestión, seguido de los grandes trazos de un marco conceptual para el análisis del trabajo de cuidados en la segunda parte. Al armazón teórico le acompañan en un tercer apartado los resultados alcanzados en el trabajo de campo, seguido de un cuarto y último de conclusiones finales.

Los resultados obtenidos han sido fruto de un estudio de corte cualitativo basado en dos recursos técnicos. Por un lado, se realizó un análisis documental del objeto del estudio, a partir de la revisión de literatura. Por otro, se empleó la técnica de la entrevista en profundidad, de tipo semiestructurada, y en la que se partió de un guion previo para canalizar las respuestas de los entrevistados. Se realizaron once entrevistas de algo más de una hora de duración cada una, grabadas en audio para su posterior transcripción. Éstas se dirigieron a personas con un amplio y profundo conocimiento sobre la temática, y que, por el ejercicio de su trabajo, cubren un abanico muy diverso y heterogéneo de familias. De tal forma que se obtuvo una información de calidad que de otra manera hubiese sido más costosa de recolectar. Acudir a familias con personas en situación de dependencia y/o a personas que asumen directamente tareas de cuidado exigiría un trabajo de campo más extenso y, especialmente, una categorización de las familias en función de múltiples factores. Así pues, se escogieron a actores clave del sector de la dependencia, fundamentalmente trabajadores sociales y representantes de Asociaciones que trabajan con el colectivo ${ }^{3}$ (ver anexo, tabla 1). Las entrevistas se realizaron a lo largo de 2015, y el ámbito territorial estudiado fue el Área Metropolitana de València.

\section{UN MARCO CONCEPTUAL PARA EL ANÁLISIS DEL TRABAJO DE CUIDADOS}

Para definir el trabajo de cuidados partiremos de la concreción del concepto trabajo. En este texto se entiende por tal la realización de actividades humanas (esfuerzo humano) cuya finalidad es la satisfacción de las necesidades de las personas mediante la producción de bienes y servicios, unas tareas que se desarrollan en contextos sociales concretos y específi-

\footnotetext{
${ }^{8} \mathrm{Su}$ selección responde a varios motivos: en primer lugar, los representantes sindicales forman parte de una plataforma cívica que actúa en defensa y representación de los intereses de las personas en situación de dependencia, al tiempo que disponen de servicios de asesoría en esta materia para las familias afectadas; en segundo lugar, los trabajadores sociales de los servicios municipales cuentan entre los cometidos principales propios de sus puestos de trabajo con el análisis acerca de las condiciones vitales (y también las laborales) de las familias, y dar respuesta a la problemática de éstas. Además, abarcan a un amplio espectro de sujetos que requieren de sus servicios, entre los que se hallan multitud de tipologías de unidades de convivencia. Y por último, los trabajadores sociales de las Asociaciones entrevistadas, nuevamente, son conocedores de la realidad vital y laboral de las familias que a ellas acuden. Sin olvidar que son Asociaciones que representan a un considerable porcentaje de personas en situación de dependencia.
} 
cos, los cuales suponen un marco esencial para la comprensión de los fenómenos laborales (Pahl, 1991: 166; Banyuls et al. 2005: 22). En esta acepción amplia cabe diferenciar dos grandes tipologías de trabajo: el trabajo mercantil y el reproductivo. El primero comporta una contraprestación económica por el esfuerzo realizado. Mientras, el trabajo reproductivo abarca al conjunto de actividades en las que no existe retribución alguna, como pueden ser, entre otras, las actividades de cuidado del hogar y de la familia (Carrasquer et al. 1998: 95), y que a su vez se caracterizan por su feminización e invisibilidad. En esta última categoría quedan recogidas acciones como las labores del hogar o el cuidado de personas, sean niños, ancianos, enfermos o personas en situación de dependencia. Y aunque el trabajo reproductivo sea un concepto más amplio que el trabajo doméstico (Carrasquer et al. 1998: 96), en aquellos trabajos circunscritos al hogar y a la familia se pueden equiparar ambos (Torns, 2008: 58).

El trabajo reproductivo es objeto de una menor valoración social, y con frecuencia también académica, que el mercantil. Con todo, hay excepciones que destacan tanto la interconexión entre ambas formas de trabajo, como la miopía de aquellas corrientes que infravaloran el protagonismo de las tareas no remuneradas, sin las que no se podría garantizar el mantenimiento de la fuerza de trabajo y la supervivencia de las generaciones presentes, donde se incluyen las tareas de organización del hogar o las que atesoran una fisionomía emocional (Torns, 2008: 58). Parte de este cambio de perspectiva está en deuda con las reivindicaciones feministas y el debate abierto acerca del trabajo productivo-reproductivo, especialmente en la década de los setenta (Parella, 2003: 29-32). La incorporación de la mujer al mercado laboral, los cambios en los modelos familiares y la mayor oferta de bienes y servicios en el mercado que podían ser sustitutos de los producidos en el ámbito del hogar, definen el escenario idóneo para este debate (Carrasco, 2006: 42) y espolean la ruptura con la norma social europea posterior a la II Guerra Mundial. La década de los ochenta abre nuevos campos de posibilidades al quebrarse el esquema producción-reproducción e incrementarse la interrelación entre ambos ámbitos (Humpries y Rubery, en Carrasco, 1994; Picchio, 1992 en Carrasco, 2006; Hernández, 2007: 181). No obstante, y según Carrasco (2006: 45), este argumento "no permitía escapar de la dimensión mercantil patriarcal”. En la actualidad, se busca alcanzar la visibilización de la contribución efectiva de hombres y mujeres a la satisfacción de las necesidades humanas, tanto vía trabajo mercantil como reproductivo, al tiempo que combatir los distintos factores generadores de desigualdades y asimetrías sociales, como las asimetrías de género.

Junto a esta ampliación del concepto trabajo, el análisis de los fenómenos laborales y otras cuestiones sociales se enriquece si incorporamos la familia como unidad de análisis, hecho destacado por la literatura (Narotzky, 1988 y 2004; Garrido y Gil, 1993; Carbonero, 1997; Wallace, 2002; Arteaga, 2007). Es en el seno de la unidad familiar donde se adoptan dos decisiones estrechamente relacionadas. Por una parte, se decide cómo se cubrirán las necesidades de sus integrantes (incluyendo las necesidades de cuidados). Se trata de concretar el recurso a alguna de las esferas antes planteadas, sea el mercado, sea el Estado (con su provisión de bienes y servicios), sea el Tercer Sector o sea la familia, y en este último caso a través 
del trabajo reproductivo (por parte generalmente de las y los miembros del hogar). Lógicamente, el recurso al mercado requiere de ingresos familiares, generalmente obtenidos a través de la incorporación de algunos de sus miembros al mercado laboral. Por otra parte, y paralelamente, se decide cómo se distribuye la carga de trabajo reproductivo y mercantil dentro de la familia. A este respecto, se debe designar quién o quiénes se encargan de las tareas domésticas, de cuidados, así como quién o quiénes se incorporan a las actividades laborales remuneradas. Estas decisiones estarán condicionadas, entre otros factores, por las posibilidades reales de mercantilizar el trabajo efectuado en el seno del hogar o el papel que desempeñen las políticas públicas en pro de mercantilizar o desmercantilizar el trabajo doméstico.

Es en este contexto en el que cabe ubicar las estrategias laborales de las familias analizadas por María Antonia Carbonero (1997), quien distingue las diferentes modalidades recogidas en la tabla 2 (ver anexo).

Se desprende, pues, que la división sexual de roles en el hogar conduce a una división sexual del trabajo y a una desigualdad/discriminación de las mujeres en el mercado laboral. $\mathrm{Al}$ asignársele a la mujer (mayoritariamente) las responsabilidades domésticas, con base en la normativa sociosexual o "normas de género" (Badget y Folbre, 1999), la participación femenina en el mercado de trabajo se ve dificultada. Al no existir una responsabilidad compartida entre hombres y mujeres, si esta última quiere participar en el mercado de trabajo, al unísono debe seguir cumpliendo con sus tareas del hogar. A su vez, las mujeres incorporadas a un mercado laboral segmentado han tendido a concentrarse en determinadas ocupaciones y empleos, por lo general, en el estrato secundario. En el primer caso, agrupándose en ocupaciones consideradas como “femeninas”, y en el segundo, quedando relegadas tanto a empleos a tiempo parcial que permitan compatibilizar hogar-empleo como a aquellos donde la temporalidad y la inestabilidad son frecuentes. De un modo u otro, gran parte de las mujeres incorporadas al mercado laboral ven cercenadas sus posibilidades de seguir una trayectoria laboral ascendente (Rodríguez y Larrañaga, 2003: 6; Rodríguez Enríquez, 2015: 34). Y con la crisis, estas incorporaciones femeninas al mercado de trabajo se han intensificado por motivos diversos, sin que se rompa con las tendencias apuntadas de fuerte presencia en los empleos precarios y gran contribución al trabajo no remunerado por parte de las mujeres (Aguado y Pitxer, 2013).

Por último, nos centraremos en los trabajos de cuidados, que recogen todas aquellas tareas con las que "afrontar y dar respuesta a las necesidades de cuidados y bienestar cotidianos de las personas, que el sistema socioeconómico capitalista o bien no afronta o bien no resuelve de manera satisfactoria” (Torns et. al., 2012: 96-97). Se trata de un trabajo que no sólo cabe reducir al ámbito del hogar sino que puede hacerse efectivo a través del mercado y/o a través de políticas públicas (incluso el voluntariado o la sociedad civil). Ligando trabajo de cuidados y Estado del bienestar emerge el "social care" u organización social del cuidado, desarrollado por Daly y Lewis (2000: 285), definido como "el conjunto de actividades y las relaciones que intervienen en la satisfacción de las necesidades físicas y emocionales de las personas adultas dependientes (...) y los marcos normativos, económicos y sociales en 
los que aquéllas se asignan y se desarrollan”. Tepo Kröger (2001: 4), a partir del Proyecto SOCCARE, incorporó a este concepto la posibilidad de ser un trabajo asalariado (o no), realizado por profesionales (o no), y que puede efectuarse tanto en la esfera pública como en la privada.

Reparando sobre el trabajo de cuidados en los hogares, sus principales rasgos en la mayoría de los casos son la invisibilidad, la no remuneración y el desempeño por el sexo femenino (Hernández, 2009: 176), cuyo sostén es el mantenimiento del orden patriarcal y androcéntrico que relega a la mujer al ámbito doméstico y a la realización de las actividades propias en éste. Asimismo, también cabe subrayar que estos trabajos revisten ciertas particularidades, en tanto que en estos cuidados están presentes dos dimensiones: una objetiva, que incluye las actividades que satisfacen las necesidades de carácter biológico, y otra subjetiva o emocional. En cuanto a las primeras, pueden ser cubiertas a través de la compra de esos cuidados en el mercado. En cambio, las atenciones de carácter subjetivo o emocional entrañan más complejidad. Si bien pueden aparecer contextos en los que el mercado puede ofrecer el componente emocional, frecuentemente esto no es posible (Carrasco, 2003: 19-20).

Por tanto, la crisis de los cuidados se ha desencadenado, además de por los motivos antes señalados, por factores como: a) la dejación de funciones por parte del Estado a la hora de satisfacer esas necesidades (Morcillo et al., 2010: 200); b) la pérdida del tejido social y la creciente precariedad, que conduce a una mayor necesidad de atenciones o cuidados; y c) las desigualdades sociales y diferencias en los niveles de vida, que marcan la senda hacia la segmentación entre quienes pueden adquirir esos cuidados en el mercado y quienes no tienen esta opción y deben recurrir a otras vías (Del Río y Pérez Orozco, 2004 en Pérez Orozco, 2006: 20). Así, las familias de clase baja no pueden acceder a la compra de servicios de cuidados en el mercado al carecer de ingresos suficientes para la contratación, quedándoles el recurso a los servicios públicos, al Tercer Sector o a la familia, siendo en este último supuesto donde el sexo femenino se ocupará de ello, sobre la base de la cultura dominante que asocia cuidados-mujer.

En definitiva, la crisis de los cuidados, que nace a partir de la reordenación y reorganización de los cuidados en aras de cubrir las (nuevas) necesidades poblacionales, supone un reto en el sistema para introducir la perspectiva de la sostenibilidad de la vida como categoría central sobre la que graviten las distintas esferas de actividad: productiva y reproductiva (Pérez Orozco, 2006: 10; Pérez Orozco, 2011: 32). Se ahonda en la pregunta sobre cómo satisfacer las necesidades humanas y sobre qué vida queremos vivir, sin que la respuesta remita a un campo de opciones que quede restringido al ámbito del mercado (Carrasco, 2006: 45; Pérez Orozco, 2011: 32; Rodríguez Enríquez, 2015: 32). Es más, para una gran parte de familias, las de clase baja, el mercado no forma parte de su campo de opciones. 


\section{UNA APROXIMACIÓN A LOS TRABAJOS DE CUIDADOS EN EL ÀREA METROPOLITANA DE VALÈNCIA}

En este tercer bloque presentaremos los principales resultados del trabajo de campo realizado. Si bien el estudio de caso se ha centrado en el Àrea Metropolitana de València, la situación identificada y las conclusiones obtenidas son extrapolables a las áreas urbanas del conjunto del País Valenciano y del Estado español.

\subsection{Modalidades de cuidados a las que se recurre: protagonismo central de la familia}

A las personas entrevistadas se les consultó sobre el abanico de alternativas de provisión de cuidados, que incluye el recurso a la familia, al Estado, al mercado o al Tercer Sector. Las respuestas marcaron claramente que el cuidado familiar es el predominante, con algunas variables que pueden incidir en la elección, básicamente la edad de la persona en situación de dependencia y el tipo y estado de la enfermedad o discapacidad de la persona dependiente. En cuanto a la edad, todos declararon que cuando son menores, el cuidado se efectúa directamente por la familia en el ámbito doméstico

“Cuando son personas dependientes menores no hay duda que el menor está en casa con la familia y con todos los cuidados...” $\left(\mathrm{E}_{2}\right)$.

Mientras, si el estado de dependencia derivaba de accidente o enfermedad sobrevenida, se presentaban más alternativas: i) cuidado en el hogar por parte del cónyuge o pareja (si lo hubiere), los ascendientes o descendientes de éste, pero en cualquier caso, remarcando que la mujer era la encargada de su desempeño (madres o hijas del dependiente, respectivamente); ii) cuidados en el ámbito doméstico por personal remunerado; o iii) cuidados extra-domésticos. En el supuesto de dependencia sobrevenida gravita como elemento destacable la edad de la persona afectada, pero sin romper el esquema básico que acabamos de describir. Como ejemplo ilustrativo el caso de accidentes de tráfico que impiden las actividades básicas de la vida diaria.

"Para gente joven, menores de treinta [años] pongamos...siempre va a ser la madre del afectado la que deje de trabajar.... A partir de los treinta [años] para arriba, va a ser la mujer del afectado o la madre del afectado quien se vaya a ocupar del cuidado. Es decir, las mujeres suelen dejar de trabajar, pero los maridos no suelen dejar de trabajar” $\left(\mathrm{E}_{4}\right)$.

\subsection{Factores decisivos para la elección de la modalidad de cuidados: un Estado del} Bienestar infradesarrollado acompañado de otros condicionantes económicos y culturales

La elección de una u otra modalidad viene condicionada por factores económicos, culturales o geográficos. Todos los entrevistados establecieron la capacidad económica como elemento decisivo a la hora de elegir uno u otro tipo, y así se recoge en diferentes fragmentos discursivos. 
"Otro factor es que tengas suficiente capacidad económica para contratar a una persona... la [familia] que tiene posibilidades económicas se nota...el problema viene con las [familias] que no tienen, y con lo poco que tienen, se tienen que acomodar...” $\left(\mathrm{E}_{4}\right)$.

"[La capacidad económica] supone la capacidad de decidir... o sea, tú puedes elegir. Si tú tienes capacidad económica, tú puedes elegir dónde estar (...) hay mucha gente que no querría ir a la residencia (...) si aquí mucha gente tuviera capacidad económica, hay mucha gente que contrataría a alguien para que le echara una mano....y se tienen que conformar con una ayuda a domicilio...” $\left(\mathrm{E}_{9}\right)$.

En un mismo nivel de importancia, un segundo ingrediente destacado para la elección de la modalidad es de tipo cultural, acompañado de un tercero consistente en el poco esfuerzo destinado a la creación de una oferta de servicios públicos de atención a la dependencia. Algo coherente con el modelo de Estado del bienestar mediterráneo, que se caracteriza, entre otros, por un peso muy elevado de la institución familiar y una estructura de atención social pública escasa, con influencias mutuas entre éstas: al no existir unos servicios sociales públicos que atiendan esta demanda, es la familia quien debe encargarse de esta atención (Naldini y Guerrero, 2009). Como muchos autores han venido resaltando (Ferrera, 1996; Rhodes, 1996; Moreno, 2002, 2006, Moreno y Marí-Klose, 2013), parte de esta falta de desarrollo de políticas sociales y de una caracterización propia de los países de la vía mediterránea se debe a un Estado del Bienestar infradesarrollado consecuencia de una industrialización tardía, de las redes clientelares, de una caracterización político-institucional propia o de un mercado de trabajo con rasgos propios: elevadas tasas de temporalidad, precariedad laboral, fortaleza de sectores productivos con bajo valor añadido o "debilidad" tecnológica.

“(...) El factor cultural, a nosotros aquí, y más en la zona nuestra que somos mediterráneos totales....el factor cultural, la afectividad, los vínculos afectivos, y el traslado de mi madre a una residencia yo lo haré cuando ya no puedo más pero sino no, porque ¿cómo voy yo a dejar a mi madre que me ha criado, me ha dado tanto...?” $\left(\mathrm{E}_{8}\right)$.

Junto a los dos factores anteriores, también se aprecia la influencia de lo que podríamos denominar factor generacional, en el sentido de que la verbalización de las personas jóvenes con familiares en situación de dependencia difiere notablemente de las generaciones anteriores, apostando los primeros por los cuidados formales (aquellos distintos al trabajo reproductivo en el seno familiar).

Para finalizar, cabe incluir como factor causal crucial la falta de recursos públicos, un factor estructural que se ha intensificado con las políticas de recortes desde la segunda mitad de 2010, lo que conduce necesariamente al cuidado en el ámbito doméstico o a que se deba acudir al mercado para cubrir esa necesidad ${ }^{4}$. La carencia de dichos recursos, que para to-

\footnotetext{
${ }^{4}$ Se trata de un nuevo impulso a un proceso de más largo alcance iniciado con las políticas de corte neoliberal por las que se optó a partir de los años ochenta. Como señala Diane Sainsbury (1996: 198 y ss), se trata de un recorte al Estado del Bienestar que, más allá de la reducción en las partidas de gasto público social, consiste en una reestructuración del mismo dirigida hacia su residualización. Esta implica "ampliar el rol del mercado en la provisión de bienestar, y restringe la provisión pública a una mínima red de seguridad en aquellos casos de fallo del mercado y no respuesta familiar" (1996: 198).
} 
dos los entrevistados es palmaria, va de la mano de la ubicación geográfica, pues las zonas rurales presentan menos posibilidades de elección. En cualquier caso, hay una gran carencia de servicios públicos de atención al colectivo. Como consecuencia de la falta de estos, son las familias quienes tratan de remediar este déficit mediante la creación de asociaciones de afectados y su provisión a través de las mismas. Y esto último va de la mano de una pequeña pero creciente presencia del Tercer Sector de Acción Social, especialmente en la época de crisis iniciada a finales de 2007 (Homs, 2009; FOESSA, 2014; Ruíz Vilafranca, 2015).

\subsection{Sobre el quién en las tareas del cuidado: omnipresencia de la mujer}

El cuidado es una tarea que, históricamente, queda atribuido a las mujeres, entrando en juego la cuestión de género. Todos los actores afirman que es la mujer quien desempeña las actividades propias del cuidado. Tareas que no sólo permanecen en el imaginario para el cuidado mediante el trabajo reproductivo en el seno familiar, sino que incluye el supuesto de externalización del cuidado.

“Mujeres, madres, el género femenino” $\left(\mathrm{E}_{6}\right)$.

“La mujer será la que ejercerá el papel de cuidadora principal” $\left(\mathrm{E}_{7}\right)$.

Efectivamente, esto también sucede cuando se recurre a la contratación de una persona para el desempeño de las tareas del cuidado en el ámbito doméstico. En este caso son las mujeres quienes copan mayoritariamente la oferta del sector en el mercado de trabajo, situación que viene alimentada por el hecho de que los demandantes marcan como preferencial el sexo femenino.

"El perfil son mujeres, sobre todo mujeres, hombres hay poquísimos...sobre todo, extranjeras. Ha habido una época que ha habido muchísimas latinoamericanas...” $\left(\mathrm{E}_{7}\right)$

Al unísono, los servicios públicos de atención a la dependencia, prácticamente ausentes en los discursos del trabajo de campo, también son un sector feminizado.

El hecho de que sean ellas las encargadas del cuidado viene determinado por los roles asignados socialmente a la mujer. A éstas se les han atribuido unas tareas como si fuesen innatas, cuando en realidad todo es fruto de una construcción o imaginario que se ha creado. Al sexo femenino se le impone, con base en las “normas de género" (Badgett y Folbre, 1999) y a partir del proceso de socialización, unos quehaceres y/o actitudes que se convierten en estereotipos y que acaban formando parte de su ser ${ }^{5}$, entre los que se hallan los vinculados con el trabajo de cuidados.

"Normalmente, por mucho que nos queramos dar de modernos, en España todavía las labores de la casa recaen en mucho grado sobre la mujer, y aunque trabajen fuera de casa. Y si no sobre la mujer...vamos a poner que trabajen los dos, también se suele

${ }^{5}$ En este sentido, ese proceso socializador aparece ejemplarmente recogido en la frase "no se nace mujer, se llega a serlo" (Beauviour, [1949]1969: 109). 
contratar a una mujer para que atienda. Que atienda a la casa y a la persona dependiente" $\left(\mathrm{E}_{3}\right)$.

\subsection{Influencia de las tareas del cuidado sobre la participación en el mercado laboral: menor movilización laboral y mayor precariedad laboral para las mujeres}

La existencia de algún miembro de la familia en situación de dependencia tiene repercusiones sobre la situación laboral del resto de integrantes de la misma. Las decisiones que deben tomar estas personas a la hora de armonizar el empleo con las tareas del cuidado son variadas, y los efectos diferirán según la composición y el sexo de cada uno de los miembros de la familia, del poder adquisitivo para adquirir esos servicios en el mercado o de la existencia de una oferta pública suficiente que pueda satisfacerlos.

Cuando existen personas dependientes, la más perjudicada en la familia desde la perspectiva del mercado de trabajo acostumbra a ser la mujer. Al asumir ésta la mayor parte de la carga de atención a la o las personas dependientes, o bien se retira totalmente del mercado de trabajo o bien se ve abocada a asumir formas de empleo precarias, en especial a través de jornadas parciales o atípicas. Y aunque es algo generalizado, se trata de un fenómeno que tiene lugar con mucha mayor intensidad en las familias de escasos recursos, en las que no existe la posibilidad de mercantilizar estos trabajos de cuidados.

"Cambiar de empleo o de tipo de jornada, sobre todo lo veo con hijos dependientes (...) En los niños o sí o sí, generalmente, suele ser la mujer la que deja el trabajo, la que cambia el turno, la que sacrifica su vida laboral para dedicarse....” $\left(\mathrm{E}_{2}\right)$.

“(...) es muy normal pasar de una jornada completa a una parcial...” $\left(\mathrm{E}_{3}\right)$.

"Pues [la mujer] posiblemente o no trabaja o se pide reducción o se busca un trabajo para poder compatibilizar, como puede ser un menjador [comedor] escolar o algo de mañanas para disponer de más tiempo para cuidar” $\left(\mathrm{E}_{8}\right)$.

Incluso, al no poder contratar a una persona para el cuidado o no querer (o no poder) acceder a centros institucionalizados por la carencia de suficiente capacidad adquisitiva y de servicios públicos especializados, con cierta frecuencia, las mujeres deciden abandonar el mercado laboral. De hecho, cuando se aprobó la LAPAD, hubo mujeres que "renunciaron a su puesto de trabajo para dedicarse al cuidado de la persona dependiente” (E1), pues aceptaron la prestación económica por cuidados en el entorno familiar, algo que fue criticado por algún entrevistado:

“(...) eso de la "pagueta” [paguilla] para el cuidado de la persona es lo que no debería de haber sido”. $\left(\mathrm{E}_{8}\right)$.

El abandono del puesto de trabajo y posterior salida del mercado laboral, pasando a considerarse como inactiva, es otra de las decisiones que se adoptan.

"La primera etapa del niño, como va tanto al médico, pues claro, ¿qué haces? no puedes estar en un trabajo cada dos por tres [pidiendo permisos]. $O$ tienes a alguien al lado que te ayude (...) O si no tienes eso, ¿a ver cómo trabajas?... es muy difícil a trabajar...” $\left(\mathrm{E}_{3}\right)$. 
"La mayoría tiene que dejar de trabajar....” $\left(\mathrm{E}_{4}\right)$.

"Porque [la mujer] está cuidando para que otros puedan trabajar...eso sí, eso es un sacrificio. Hay gente que no busca” $\left(\mathrm{E}_{9}\right)$.

Por su parte, los actores sociales traen a colación el contenido de los convenios colectivos y la normativa reguladora que facilita la conciliación laboral y familiar. Sin embargo, y a pesar de este notable avance "al final concilia quien concilia, cómo concilia, y quien se lo puede permitir, si puedes permitirte reducir horas, si no puedes, a costa de qué, de que como me desengancho un tiempo del ámbito laboral...” (E2), que viene siendo, mayoritariamente, el caso de las mujeres.

Las repercusiones para la mujer superan lo recogido hasta el momento, pues la reducción de jornada o la opción por una excedencia perjudica tanto al salario que percibe como a las posibilidades de promocionarse, dada la interrupción de su trayectoria laboral. A lo que cabe añadir una mayor probabilidad de caer en situaciones de precariedad y, de forma adicional, una pérdida de autonomía económica, o lo que es lo mismo una dependencia económica en relación con la pareja o cónyuge (si lo hubiere). Y tiene repercusiones de más largo plazo, en tanto que unos menores salarios conllevan inferiores cotizaciones sociales y, a su vez, una reducción en las pensiones de jubilación futuras.

“(...) al final, si pide una reducción de jornada, también hay una repercusión económica, cobra menos... (...) o dejan su trabajo, entonces pierden el lazo con el mercado laboral, o si vuelven tienen que contratar a alguien que cuide de su pareja, y en ocasiones, no vuelven con todas las horas, ni siquiera el acceso....porque claro, eso significa "salir corriendo" a veces irte a determinadas horas...por lo tanto, posiblemente pierdan la posibilidad de ascender...sí, es verdad, cambia... " $\left(\mathrm{E}_{6}\right)$.

“(... ) Tengo menos posibilidades de promoción, el jefe cuenta menos conmigo...” $\left(\mathrm{E}_{2}\right)$.

En consecuencia, lo hasta ahora expuesto es coherente con el enfoque de la segmentación de los mercados de trabajo y su visión multicausal explicativa. Según esta perspectiva, existe un mercado de trabajo secundario, caracterizado por la existencia de salarios más bajos, peores condiciones laborales, inestabilidad en el empleo y una elevada rotación involuntaria (Recio, 1991, 1999; Banyuls et al., 2005; Rubery, 2007; Fernández-Huerga, 2010). A estos puestos de trabajo son relegadas determinadas clases sociales, etnias o razas, así como jóvenes y mujeres, todos ellos con escaso poder de negociación. Pue bien, ese mercado secundario es crucial desde la perspectiva de los trabajos de cuidados. Por un lado, porque la mayor parte de estos trabajos de cuidados remunerados se ubican en el mercado de trabajo secundario, y por otro, porque aquellas mujeres con una sobrecarga de trabajo de cuidados en su familia y que no abandonan el mercado laboral se ven relegadas a empleos precarios. 


\subsection{Cambios en el peso de cada uno de los ámbitos de cuidados: menor provisión pú- blica y revitalización del resto de opciones}

En el marco de residualización del Estado del Bienestar que citaba Sainsbury (1996), el empeoramiento de la situación económica desde finales del 2007 ha activado un conjunto de factores que empujan a las familias a modificaciones o cambios intensos en el peso y distribución de los ámbitos del cuidado y a la articulación entre estos. Son varios los cambios a este nivel.

Así, en las entrevistas se declara que se ha producido un retorno al hogar de los dependientes o una refamiliarización (o desmercantilización) de los cuidados, dado que las familias no pueden hacer frente al pago de centros de día o residencias. E incluso, con el aumento de las tasas de paro y un mayor número de familias con alguno de sus miembros en situación de desempleo, la solución discurre por hacer uso de la pensión o la prestación de la persona en situación de dependencia y así cubrir los gastos de la unidad familiar. Esto se produce tanto en el caso de personas ancianas con necesidad de asistencia como en cualquier otra franja etaria o por causa de dependencia sobrevenida. Esta opinión es compartida por todos los informantes, como muestra el siguiente fragmento:

“...hay gente que ha sacado a sus enfermos de sus residencias, porque están viviendo con el dinero de las pensiones (...) «yo no puedo meterlo en una residencia porque estamos viviendo de la pensión de mi madre»" $\left(\mathrm{E}_{7}\right)$.

Por otra parte, además de esta refamiliarización, otra opción ha sido la de prescindir de la persona contratada que desarrollaba sus tareas en la esfera doméstica o la reducción de su jornada laboral, todo y pese a que es una modalidad más económica para las familias, presentándose como causa principal la falta de capacidad económica para retribuir a esa trabajadora.

"Sí se ha desinstitucionalizado, y (...) también que a lo mejor antes tenían un cuidador, que podría ser un inmigrante, contratado...o apalabrado, y que ahora lo han dejado y ya son ellos mismos [la familia] porque con ese dinero distribuyen para toda la familia” $\left(\mathrm{E}_{6}\right)$.

“....o le ha bajado las horas (a la persona contratada)...normalmente le han bajado las horas...” ( $\left.\mathrm{E}_{8}\right)$.Otra alternativa es que, en aquellos supuestos en los que una persona de la familia se ha quedado en situación de desempleo, se decide que sea ésta quien desempeñe las tareas de cuidado, bien por disposición de tiempo libre para hacerlo, bien porque se le abona a ella la remuneración que hasta el momento estaba recibiendo la personas contratada.

Para finalizar, otra derivada es que en aquellos casos en los que se opta por la contratación de una trabajadora para el cuidado doméstico ya no sólo hay personas procedentes de otros países, mayoritariamente mujeres latinoamericanas, sino que se ha (re)incorporado a esta actividad de manera significativa la mujer nativa/autóctona, que aquí denominamos (re) nativización. 


\begin{abstract}
"Ahora el trabajo que antes sólo hacían los inmigrantes ahora lo hacen también mujeres nacionales...ahora hay españolas (...) ahora hay casos de familiares de afectados que te dicen «oye, mira, si alguien necesita un cuidador, decídmelo, que mi prima, que mi hijo o que tal, necesita trabajo y no sé cuántos»” $\left(\mathrm{E}_{5}\right)$.

"Hombre, pues si me hubieras hecho esta pregunta hace unos años, te diría cualquier persona latinoamericana, pero ahora ya hay muchas mujeres españolas que cuidan” $\left(\mathrm{E}_{4}\right)$.

"En cuanto a la nacionalidad hasta hace unos años, eran casi un 100\% extranjeras, pero desde la crisis la cosa ha cambiado, ahora ya hay más presencia de mujeres nativas....y también de Europa del Este, pero en menor porcentaje” $\left(E_{1}\right)$.
\end{abstract}

A todo lo anterior cabe añadir el recurso creciente a Asociaciones comprendidas dentro del Tercer Sector de Acción Social. Éste, sin haber incrementado los recursos, ha ampliado el número de personas atendidas en la actual época de crisis y dificultades.

\title{
4. CONCLUSIONES
}

La visión y representación ofrecida por los informantes clave en torno a los objetivos fijados en este texto se concentran en que a) el cuidado en el ámbito doméstico por los miembros de la familia es el más frecuente, aunque ni puede generalizarse a todas las familias ni supone la total desaparición del recurso a otras alternativas de provisión de cuidados; b) los factores que mayor repercusión tienen a la hora de decidir qué modalidad de cuidado se escoge son, junto con el condicionante que supone la opción política dirigida a recortar el Estado del Bienestar en el sentido de residualización de éste, los de cariz económico o capacidad adquisitiva de las familias y los de tipo cultural, estos últimos ligados a las características propias de los países incluidos dentro de la "vía mediterránea”; c) es la mujer quien se encarga de la realización de las tareas del cuidado, más allá de la edad de ésta, aunque parece existir alguna diferencia intergeneracional; d) dado que son las mujeres quienes desempeñan estas tareas, son ellas quienes deben enfrentarse a la difícil misión de compatibilizar la vida laboral con la familiar, y dentro de esta última, los cuidados de la persona en situación de dependencia; e) las respuestas en este último ámbito, al final, repercuten de forma negativa en la situación y trayectoria laboral de las mujeres, que se ven abocadas a la precarización y, en definitiva, a verse encasilladas en el mercado de trabajo secundario; f) existe una carencia de servicios públicos suficientes para satisfacer las necesidades de las personas en situación de dependencia, a pesar de que exista legislación que lo promueva. Esta carencia repercute tanto en la persona afectada (por la falta de servicios que faciliten su autonomía y/o la protección ante situación de dependencia) como en la unidad familiar, y de forma sobresaliente sobre la mujer, que es la que se encarga de los cuidados en la esfera doméstica. La escasez de servicios públicos cuyo fin sea la atención a las personas dependientes no permite a la mujer desarrollar su vida laboral en las mismas condiciones que los hombres. A su vez, g) esa carencia de derechos y servicios públicos suficientes junto al abandono del recurso al mercado, consecuencia de la falta de capacidad económica suficiente derivada de la crisis de finales del 2007 y de las políticas de austeridad, han facilitado 
el tránsito a la refamiliarización de los cuidados y a la búsqueda de alternativas mercantiles más económicas, con bastante frecuencia en un contexto de informalidad y que se ha traducido en la (re)nativización o retorno de la mujer nativa/autóctona a estas actividades de forma remunerada. Por último, h) esta refamiliarización se ha visto acompañada del apoyo en el Tercer Sector de Acción Social por parte de un número creciente de familias. Si bien éste es un ámbito que no tiene un protagonismo destacado en la atención a la dependencia, su peso relativo ha crecido en la última época de crisis.

En suma, la falta de un desarrollo suficiente de derechos y de provisión pública en el ámbito de los cuidados, la intensificación de los recortes (en un sentido general) en los últimos años, y la opción por unas políticas laborales desreguladoras/flexibilizadoras y un mercado laboral crecientemente precarizado, han propiciado el desarrollo de las alternativas de provisión no públicas. Esto es algo que hemos podido constatar en el Àrea Metropolitana de València, territorio integrado en una comunidad autónoma en la que las políticas y el gasto social no han sido una prioridad en las últimas décadas de gobiernos conservadores, con nefastas consecuencias sociales y laborales, en especial para las familias con menores recursos y para las mujeres en general. Urge, pues, una recuperación de derechos sociales y laborales.

\section{BIBLIOGRAFÍA}

Aguado, T.E. y Pitxer, J.V. (2013). Incidencia de la crisis y el desempleo sobre las mujeres, I Congreso Estatal de Centros Universitarios de Ciencias del Trabajo y Relaciones Laborales, València 12 y 13 de septiembre de 2013.

Arteaga, C. (2007). Pobreza y Estrategias Familiares: Debates y Reflexiones, Revista Mad: Revista del Magíster en Análisis Sistémico Aplicado a la Sociedad, nº 17, pp. 144-164.

Badgett, M.V. L. y Folbre, N. (1999). ¿Quién cuida de los demás? Normas sociosexuales y consecuencias económicas”. Revista Internacional del Trabajo, vol. 118 (3), pp. 347-365. Doi: 10.1111/j.1564-913X.1999.tb00137.x

Balbo, L. [1978](1994). La doble presencia en Borderías, C., Carrasco, C. y Alemany, C.(eds) Las mujeres y el trabajo: rupturas conceptuales. (pp. 503-514), Barcelona: Ed. Icaria.

Banyuls, J., Cano, E, Pitxer, J.V. y Sánchez, A. (2005). Economia laboral i polítiques d'ocupació. València: PUV.

Beauvoir, S. [1949](1969). El segundo sexo. Buenos Aires: Siglo Veinte.

Benería, L. (1999). El debate inconcluso sobre el trabajo no remunerado, Revista Internacional del Trabajo, vol. 118(3), pp. 321-346. Doi: 10.1111/j.1564-913X.1999.tb00136.x

Carbonero, M.A. (1997). Estrategias Laborales de las Familias en España. Madrid: Consejo Económico y Social. 
Carrasco, C. (2003). ¿Conciliación? No, gracias. Hacia una nueva organización social, en Amoroso, M.A., Bosch A., Carrasco, C., Fernández, H., Moreno, N. (eds) Malabaristas de la vida. Mujeres, tiempos y trabajos. (pp. 16-34) Barcelona. Ed. Icaria- Disponible en http://www.caladona.org/wp-content/pujats/2009/12/2003-malabaristas-de-la-vida-DONES-I-TREBALLS.pdf

Carrasco, C. (2006). La paradoja del cuidado: necesario pero invisible, Revista de Economía Crítica, $n^{\circ}$ 5, pp. 39-64.

Carrasco, C. (2011). La economía del cuidado: planteamiento actual y desafíos pendientes, Revista de Economía Crítica, nº 11, primer semestre, pp. 205-225.

Carrasquer, P., Torns, T., Tejero, E. y Romero, A. (1998). El trabajo reproductivo, Papers, $n^{o}$ 55, pp. 95-114.

Daly, M. y Lewis, J (2000). The concept of social care and the analysis of contemporary welfare states. British Journal of Sociology, vol 51, (2), pp. 281-298. DOI: 10.1111/j.14684446.2000.00281.x

España (2006). Ley 39/2006, de 14 de diciembre, de promoción de la autonomía personal y atención a las personas en situación de dependencia. Secretaría de Estado de Servicios Sociales, Familias y Discapacidad.

Esping-Andersen, G. [1990](1993). Los Tres mundos del Estado del Bienestar. València: Edicions Alfons el Magnànim.

Fernández-Huerga, E. (2010). La teoría de la segmentación del mercado de trabajo: enfoques, situación actual y perspectivas de futuro. Investigación económica, vol 69(273) Disponible en http://www.scielo.org.mx/scielo.php?script=sci_arttext\&pid=S0185-16672010000300004

Ferrera, M. (1996). The Southern Model of Welfare in Social Europe, Journal of European Social Policy, 6(1), pp. 17-37. Doi: 10.1177/095892879600600102

FOESSA (2014). VII Informe sobre exclusión y desarrollo social en España, Madrid: Fundación FOESSA y Cáritas Española. Disponible en http://www.foessa2014.es/informe/uploaded/descargas/VII_INFORME.pdf

Garrido L. y Gil E. (1993). El concepto de estrategias familiares en Garrido, L. y Gil E. (eds.), Estrategias familiares (pp. 13-34) Madrid: Alianza Editorial.

Hernández, A. (2009). El trabajo no remunerado de cuidado de la salud: naturalización e inequidad, Revista Gerencia y Políticas de Salud, $n^{\circ} 8$ (17), pp. 173-185.

Homs, O. (2009). Los retos del Tercer Sector ante la crisis, Madrid: Fundación Luis Vives. 
Humpries, J. y Rubery, J. (1994). La autonomía relativa de la reproducción social: su relación con el sistema de producción en Borderías, Cristina, Carrasco, Cristina y Alemany, Carme (eds): Las mujeres y el trabajo, (pp. 393-424). Barcelona: Icaria.

IMSERSO (2005). Libro Blanco sobre la Dependencia. Atención a las personas en situación de dependencia en España. Ministerio de Trabajo y Asuntos Sociales.

INE (2012). Proyecciones de Población 2012. Disponible en http://www.ine.es/prensa/np744.pdf

Kröger, T. (2001). Comparative research on social care. The state of the art. SOCCARE Project Report 1. Bruselas, Disponible en http://cordis.europa.eu/documents/documentlibrary/90834291EN6.pdf

Lewis, J. (1992). Gender and the Development of Welfare Regimes. Journal of European Social Policy, vol. 2, n. 3, pp. 159-173. Doi: 10.1177/095892879200200301

Minguela, M. Á. y Camacho, J.A. (2015). Cuidados mixtos y cuidados informales a los mayores dependientes, ¿son complementarios o sustitutivos?: una visión comparada entre los países del sur de Europa. Ponencia V Congreso REPS (Red Española de Política Social). Desigualdad y Democracia: Políticas Públicas e innovación social. Barcelona. 5-6 febrero. Disponible en http://repsbarcelona2015.es/wp-content/uploads/2015/01/ Panel-09_Dependencia_MinguelaCamacho_REPS2015.pdf

Morcillo, J.M., Martín, M.C. y Ruiz, S. (2009). Mujer, mujeres, políticas de igualdad y su relación con la gestión de los cuidados. Humanismo y Trabajo Social, vol 8(9), pp. 191211.

Moreno, L. (2002). Bienestar mediterráneo y «supermujeres» .RES. Revista Española de Sociología, $n^{o} .2$, pp. 41-56.

Moreno, L. (2006). The model of social protection in Southern Europe, Revue française des Affaires sociales., 5(5), pp. 73-95. http://www.cairn.info/revue-francaise-des-affairessociales-2006-5-page-073.htm

Moreno, L. y Marí-Klose, P. (2013). Youth, family change and welfare arrangements: Is the South still so different?, European Societies. 15(4), pp. 493-513. Doi: $10.1080 / 14616696.2013 .836400$

Naldini, M. y Jurado, T. (2009). Families, Markets and Welfare States: The Southern European Model en Seventh ESPANET Conference, "The future of the welfare state, Paths of social policy innovation between constraints and opportunities”. Urbino, Italia, 17-19 septiembre.

Narotzky, S. (1988). Mujeres, hogares y talleres. València: Edicions Alfons el Magnànim - IVEI.

Narotzky, S. (2004). Antropología económica: nuevas tendencias, Barcelona: Melusina. 
Pahl, R H. [1984](1991): Divisiones del trabajo, Madrid. Ministerio de Trabajo y Seguridad Social.

Parella, S. (2003). Mujer, inmigrante y trabajadora: la triple discriminación. Barcelona. Ed. Anthropos.

Pérez Orozco, A. (2006). Amenaza tormenta: la crisis de los cuidados y la reorganización del sistema económico. Revista de Economía Crítica, $n^{\circ}$ 5, pp. 7-37.

Pérez Orozco, A. (2012). Crisis multidimensional y sostenibilidad de la vida, Investigaciones feministas, vol. 1, pp. 29-53.

Pérez Orozco, A. y Río, S. (2004). Una visión feminista de la precariedad desde los cuidados. Comisión Confederal contra la precariedad de CGT.

Picchio, A. [1984](2011). La reproducción social y la estructura básica del mercado laboral en Carrasco, C., Borderías, C. y Torns, T. (eds.): El trabajo de cuidados. Historia, teoría y políticas. (pp. 122-144), Madrid: Los Libros de la Catarata.

Recio, A. (1999). La segmentación del mercado de trabajo en España en Miguélez, F. y Prieto C.(dirs.) Las relaciones laborales en España. (pp. 125-150), Madrid: Siglo XXI.

Rhodes, M. (1996). Southern European Welfare States: Identity, Problems and Prospects for Reform. South European Society and Politics, vol 1(3), pp.1-22. Doi: $10.1080 / 13608749608539480$

Rodríguez, A. y Larrañaga, M. (2003). El trabajo de las mujeres: claves para entender la desigualdad laboral. Formación y Acreditación en Consultoría para la Igualdad de Mujeres y Hombres.

Rodríguez, C. (2015). Economía feminista y economía del cuidado, Nueva Sociedad, $n^{\circ} 256$, pp. $30-44$.

Rubery, J. (2007). Developing segmentation theory: a thirty years perspective, Economies et sociétés, vol. 41 (6), pp. 941-964.

Ruíz Villafranca, R. (dir.) (2015). El Tercer Sector de Acción Social en 2015: Impacto de la crisis, Plataforma de ONG de Acción Social - Plataforma Tercer Sector - EEA Grants. Disponible en http://www.plataformaong.org/ciudadaniaactiva/tercersector/index.php

Sainsbury, D. (ed.) (1994). Gendering Welfare States. Londres: Sage Publications.

Sainsbury, D. (1996). Gender, Equality and Welfare States. Cambridge, New York, Melbourne: Cambridge University Press.

Torns, T. (2008). El trabajo y el cuidado: cuestiones teórico-metodológicas desde la perspectiva de género, Empiria. Revista de Metodología de Ciencias Sociales, $n^{\circ} 15$, pp. 53-73. 
Los cuidados en un contexto de crisis económica... | Óscar Muñoz González et al.

Torns, T., Borrás, V., Moreno, S. y Recio, C. (2012). El trabajo de cuidados: un camino para repensar el bienestar, Papeles de relaciones ecosociales y cambio global, $n^{\circ} 119$, pp. 93-101.

Wallace, C. (2002). Household Strategies: Their Conceptual Relevance and Analytical Scope in Social Research, Sociology, vol.36(2), pp. 275-292. Doi: 10.1177/0038038502036002003. 
Los cuidados en un contexto de crisis económica... | Óscar Muñoz González et al.

\section{ANEXOS}

Tabla 1. Perfil informantes clave

\begin{tabular}{|c|c|c|}
\hline $\begin{array}{l}\text { № } \\
\text { entrevista }\end{array}$ & Perfil del entrevistado & $\begin{array}{l}\text { Duración } \\
\text { entrevista }\end{array}$ \\
\hline$E_{1}$ & $\begin{array}{l}\text { Representante de UGT-Pais Valencià y Miembro de la Plataforma en } \\
\text { defensa de la LLei de la Dependència }\end{array}$ & $1 \mathrm{~h} 40 \mathrm{~m}$ \\
\hline $\mathrm{E}_{2}$ & $\begin{array}{l}\text { Trabajadora social de CCOO-Pais Valencià y miembro de la Plataforma en } \\
\text { defensa de la LLei de la Dependència }\end{array}$ & $1 \mathrm{~h} 20 \mathrm{~m}$ \\
\hline$E_{3}$ & $\begin{array}{l}\text { Trabajadora social en Asociación Valenciana de Dependientes y } \\
\text { Cuidadores }\end{array}$ & $1 \mathrm{~h} 20 \mathrm{~m}$ \\
\hline $\mathrm{E}_{4}$ & $\begin{array}{l}\text { Trabajadora Social y responsable en la Asamblea Local de Valencia de la } \\
\text { Cruz Roja }\end{array}$ & $1 \mathrm{~h} 40 \mathrm{~m}$ \\
\hline$E_{5}$ & $\begin{array}{l}\text { Trabajador Social y responsable de Asociación de Daño Cerebral Adquirido } \\
\text { de Valencia }\end{array}$ & $1 \mathrm{~h} 35 \mathrm{~m}$ \\
\hline$E_{6}$ & $\begin{array}{l}\text { Terapeuta ocupacional y corresponsable de la Asociación de Daño Cerebral } \\
\text { Adquirido }\end{array}$ & $1 \mathrm{~h} 25 \mathrm{~m}$ \\
\hline$E_{7}$ & Trabajadora social de la Asociación de Familiares de Alzhéimer Valencia & $1 \mathrm{~h} 10 \mathrm{~m}$ \\
\hline $\mathrm{E}_{8}$ & $\begin{array}{l}\text { Trabajadora Social de Servicios Sociales municipales del Àrea } \\
\text { Metropolitana de Valencia y Profesora de la Universitat de València }\end{array}$ & $1 \mathrm{~h} 35 \mathrm{~m}$ \\
\hline$E_{9}$ & $\begin{array}{l}\text { Trabajadora Social de Servicios Sociales municipales del Àrea } \\
\text { Metropolitana de València }\end{array}$ & $1 \mathrm{~h} 20 \mathrm{~m}$ \\
\hline $\mathrm{E}_{10}$ & $\begin{array}{l}\text { Representante de orden religiosa sita en la ciudad de Valencia que } \\
\text { desempeña labores de formación e intermediación laboral. }\end{array}$ & $1 \mathrm{~h} 05 \mathrm{~m}$ \\
\hline$E_{11}$ & $\begin{array}{l}\text { Trabajadora social de Centro de Salud municipal del Área Metropolitana } \\
\text { de Valencia }\end{array}$ & $1 \mathrm{~h}$ \\
\hline
\end{tabular}

Fuente: Elaboración propia.

Tabla 2. Estrategias laborales de las familias de Carbonero Gurmendí (1997)

\begin{tabular}{|c|c|c|}
\hline Estrategias & Tipos & Descripción \\
\hline \multirow{4}{*}{$\begin{array}{l}\text { Estrategia de especialización } \\
\text { (un solo miembro activo en el } \\
\text { mercado laboral) }\end{array}$} & Tradicional & $\begin{array}{l}\text { Marido = activo } \\
\text { Mujer = inactiva }\end{array}$ \\
\hline & Sustitutoria & $\begin{array}{l}\text { Mujer u otro miembro de la familia = activa } \\
\text { Marido = inactivo }\end{array}$ \\
\hline & Transitoria & $\begin{array}{l}\text { Hombre = activo } \\
\text { Mujer = inactiva pero con intención de } \\
\text { reincorporación al mercado laboral }\end{array}$ \\
\hline & Estable & $\begin{array}{l}\text { Hombre y mujer aceptan la división de } \\
\text { responsabilidades: hombre en empleo y } \\
\text { mujer en tareas domésticas }\end{array}$ \\
\hline \multirow{5}{*}{$\begin{array}{l}\text { Estrategia de diversificación } \\
\text { (más de un miembro activo en } \\
\text { el mercado laboral) }\end{array}$} & Asociativa & $\begin{array}{l}\text { Dos miembros de la familia en activo y } \\
\text { contribuyen simétricamente en el trabajo } \\
\text { remunerado }\end{array}$ \\
\hline & & $\begin{array}{l}\text { Dos miembros en activo. } \\
\text { Dos tipos: }\end{array}$ \\
\hline & Complementaria & $\begin{array}{l}\text { a. Tradicional: el marido desarrolla la } \\
\text { actividad principal (el trabajo } \\
\text { remunerado) y la mujer tiene un } \\
\text { peso secundario }\end{array}$ \\
\hline & & $\begin{array}{l}\text { b. No tradicional: la mujer desarrolla } \\
\text { la actividad principal (el trabajo } \\
\text { remunerado) y el marido tiene un } \\
\text { peso secundario }\end{array}$ \\
\hline & Interdependiente & $\begin{array}{l}\text { Interdependencia en relación con las } \\
\text { remuneraciones percibidas por los } \\
\text { miembros de la familia }\end{array}$ \\
\hline
\end{tabular}

Fuente: Elaboración propia. 